\title{
Automatically Determining Phishing Campaigns using the USCAP Methodology
}

\author{
Robert Layton \\ Internet Commerce Security Laboratory \\ University of Ballarat \\ Email: r.layton@icsl.ballarat.edu.au
}

\author{
Paul Watters \\ Internet Commerce Security Laboratory \\ University of Ballarat \\ Email: p.watters@icsl.ballarat.edu.au
}

\author{
Richard Dazeley \\ Data Mining and \\ Informatics Research Group \\ University of Ballarat \\ Email: r.dazeley@ballarat.edu.au
}

\begin{abstract}
Phishing fraudsters attempt to create an environment which looks and feels like a legitimate institution, while at the same time attempting to bypass filters and suspicions of their targets. This is a difficult compromise for the phishers and presents a weakness in the process of conducting this fraud. In this research, a methodology is presented that looks at the differences that occur between phishing websites from an authorship analysis perspective and is able to determine different phishing campaigns undertaken by phishing groups. The methodology is named USCAP, for Unsupervised SCAP, which builds on the SCAP methodology from supervised authorship and extends it for unsupervised learning problems. The phishing website source code is examined to generate a model that gives the size and scope of each of the recognized phishing campaigns. The USCAP methodology introduces the first time that phishing websites have been clustered by campaign in an automatic and reliable way, compared to previous methods which relied on costly expert analysis of phishing websites. Evaluation of these clusters indicates that each cluster is strongly consistent with a high stability and reliability when analyzed using new information about the attacks, such as the dates that the attack occurred on. The clusters found are indicative of different phishing campaigns, presenting a step towards an automated phishing authorship analysis methodology.
\end{abstract}

\section{INTRODUCTION}

Phishing presents a major problem for all involved in this scam. An attacker, the phisher, takes the guise of a trusted third party, such as a bank or a social website. Through this guise, they attempt to acquire confidential information about a victim in order to profit from this information. Examples of the types of information that can be stolen using this technique are bank login details; usernames and passwords; and system access codes. Other information which can be used to conduct identity theft and identity fraud are targeted in phishing attacks including a victim's birth-dates, their mother's maiden name and their address. The effects of phishing has implications far beyond these impacts on the victim, as phishing can hurt the trust that a corporation carries with its consumers. This can result in a loss of business and potential income, such as scaring bank customers away from using online banking or from a particular brand.

The problems that the phisher themselves have to deal with are unlikely to raise much empathy but are important for research into phishing scams. For an email based attack, a fraudulent email attempts to direct a user to a phishing webpage in order to collect login details, the phisher faces many problems, thanks to the efforts of researchers in the anti-phishing industry. An email must be crafted to bypass phishing and spam filtering techniques [2] but still convey enough information to make the end user think that the contact is legitimate [15]. The language must be specific without using keywords that trigger alerts [3], [31] and must contain a way to direct the user to the phishing webpage without looking suspicious. The URL of the phishing website will be different from the target corporations real website, but must be disguised to look the same. The phishing website itself must convey the branding, general appearance and interaction that makes it seem legitimate [25]. However the phishing website must then bypass phishing filters and try to determine if anyone is trying to remove it, such as a cybercrime research firm trying to poison the stolen records. Each of these problems represents a choice that the phishing attack author(s) must make in order to run a successful attack.

The issue of choice when creating a document is the theoretical basis behind authorship analysis [29]. When writing a research article, a book and even a website, there are many choices that the authors make in the creation process. By analyzing these choices, the author of a document can be inferred from previous work or by distinction from other work in the area. Classic work in this area attributes the Federalist papers to Madison rather then another claiming author in Hamilton, based on the distribution of word counts [26]. In this work, the choice of which words to use was considered different between the two claiming authors and Madison's choice of words more closely matched those of the disputed papers.

Authorship analysis can even be performed on choices within a requirement of structured text, such as source code of computer programs [27]. Despite the rigid and required language required for the compilation of computer programs, traits relating to authorship are still evident [14]. Traits such as the methods of indentation and usage of upper or lower class letters for variable names were able to discern authorship with a high accuracy [27]. A common theme in authorship analysis is that the more semantic the difference between available choices, the more that choice determine authorship. Examples include function words and structural choices which have been shown to be very effective [34]. 


\section{A. Research Questions}

In this research, a methodology for determining campaigns of phishing attacks in a reliable manner is given, called USCAP. This methodology is presented, applied to a corpus of phishing website contents and the results show the size and scope of different phishing campaigns. A campaign is considered to be a series of similar phishing attacks by the same author, where authorship of a phishing attack is described in subsection II-A. This work shows the extent of the different authors, who may or may not be directly responsible for the phishing attack, but are facilitators of the crime.

This work is motivated by the need from law enforcement agencies (LEAs) for a reliable method of determining the size and scope of the operations of different phishing groups. Through this, the aim of this work is to determine the size and scope of the authors of phishing websites, in order to estimate the more general problem of phishing groups.

In answering the below research questions, LEAs will have a methodology that is able to consistently and reliably determine the authorship of phishing websites in order to provide a target for criminal investigations. The ability to focus investigations on a single target has been identified as a potential benefit to cybercrime investigations, which are often hampered by the lack of direct authorship of attacks. Online service providers could also use this information to gain information about their adversaries. This information can be used in turn to establish more directed responses to phishing attacks, such as warning customers about key pieces of information to look for in the current attacks. This information can be determined by profiling each of the current phishing campaigns.

The work presented in here aims to answer the following research questions:

1) Does the unsupervised SCAP (USCAP) methodology provide reliable and consistent clusterings of phishing websites by campaign?

2) What is the size and scope of each phishing campaign recognized by the USCAP methodology?

\section{B. Dataset}

The dataset used for this research is a collection of over 700 phishing websites targeting a major Australian banking institution. The dataset contains the full raw phishing website source code, which was retrieved by following links in emails marked as phishing. All websites in the database have been verified as phishing by industry experts. The date a website was retrieved and the URL it was hosted on has been collected in the dataset. This extra information is used in the evaluation phase, described in subsection III-D.

\section{Authorship AnAlysis On Phishing}

Cybercrime is an important application of authorship analysis due to its increasing impact on today's lifestyle. The nature of the Internet makes anonymity easy while still allowing Internet based crime to have a large impact, resulting in a need for indirect methods of attack attribution. The types of cybercrimes that authorship analysis has been applied to vary wildly. Examples include webpage spam [33], [32]; malware [9], [7]; pornography [19]; and online terrorism postings [1]. Authorship analysis has been applied to phishing attacks as well [17], [18], presenting the basis that this research extends.

Phishing was first popularized in the mid 1990s and has since been a hot topic of research, with many research papers devoted to the problem and also conferences held, such as the eCrime Researcher's Summit which is held by the AntiPhishing Working Group. Much phishing research focuses on classification of phishing messages and websites for detection and blocking [20], [21], [3], [2]. Other work in phishing looks at phishing from a crime perspective, aiming to understand the motives of phishers [24] to understanding the organization behind phishing attacks [23]. Recent research into phishing has started to look at the authorship properties of phishing attacks.

This research takes the existing body of knowledge about authorship analysis and applies it to phishing to develop an autonomous system capable of clustering phishing websites by campaign. Previous work in phishing authorship was able to cluster together groups of phishing attacks using an in-depth analysis that was driven by expert knowledge [23]. To perform a similar analysis on new data would take almost as long as the original study, due to the investigative nature of the work. This provides the motivation behind automating this task and the justification for this requirement on this research.

In this research, a methodology is presented that is able to automatically clusters phishing attacks into different campaigns against the same target. The resulting clusters are evaluated using information not present during the training of the system to determine the validity of the clusters. The aim of this research is to provide an intermediary step between phishing attacks, and automatic phishing authorship detection. This step is the collection of phishing websites into 'campaigns' which are similar phishing attacks by the same author. A campaign should have a high precision, in that phishing attacks in the same campaign should belong to the same author. This is opposed to having a high recall, where all phishing attacks by the same author are in the same group. Future work in this area by the authors will focus on this final step, grouping campaigns into authorship clusters with both a high precision and high recall.

\section{A. A Question of Authorship}

The underground economy is a complex mix of suppliers and customers of various parts of cybercrime attacks [30], [24]. An attack needs a procedure, a distribution platform and a method of obtaining the money. There are criminals who are experts in each of these fields, for each of the various types of cybercrimes. One element of a phishing attack that is integral to this work is the phishing kit.

A phishing kits are 'complete phishing websites contained in a ready-to-deploy package'[5]. Due to the availability of both sold and free phishing kits, it is quite possible that the author of the phishing website is not the person responsible for the phishing attack. For this research, we concern ourselves 
with the author of the phishing attack which in many cases would be the person who created the phishing kit, not the person who used it. These phishing kit authors should still be considered criminals, as they profit from crimes, assist crime and are creating tools to deliberately subterfuge protection and security systems. In many countries these are seen to be almost as much of a crime as the original phishing attack. Further to this point, if LEAs are able to prosecute the authors of these phishing kits then the phishing ecosystem would break down due to the unavailability of phishing websites.

\section{B. The SCAP Methodology}

The Source Code Authorship Profile (SCAP) methodology is a methodology developed to determine authorship of computer programs by their source code [8], [7]. It was developed based on earlier work in the area of software authorship attribution [16], [14], [11], [22]. The SCAP methodology is a simplified form of another localized $n$-gram based method [13] which has a more complex distance metric.

The SCAP methodology uses character level $n$-gram distributions to generate a profile for an author. To generate the Simplified Profile (SP) for an author, all training documents for that author are combined and the frequency of each character level $n$-gram is counted. The $L$ most frequently occurring $n$ grams are then collected. These form the SP for that author and this procedure is then repeated for all known authors. To attribute a new document to a known author, the Simplified Profile Intersection (SPI) is calculated between the document and each author with the author with the highest SPI is given as the author. To calculate the SPI between a document and an author, the $L$ most frequently occurring $n$-grams for the document are collected and compared to the author's SP. The SPI is the size of the intersection of these two profiles, the number of $n$-grams appearing in both lists of $n$-grams.

The SCAP methodology differs from the earlier work of Keselj et. al [13] through the simplification of the distance metric. In [13], the frequencies of each $n$-gram are needed to calculate the Relative Distance (RD) between a document and author. This makes the profiles more complex and the distance metric also takes longer to compute. It was shown that SPI performs either better than or comparably with RD [8], giving weight to the use of SPI instead of RD for this methodology.

\section{Cluster Analysis and Validation}

In cluster analysis, the dataset is grouped into subsets such that instances within a subset are more similar to each other then to instances in other subsets.under a wide range of parameters Different clustering algorithms attempt this in different ways and many try to find a way of clustering the data to minimize a given evaluation metric. The $k$-means algorithm [12] is perhaps the most famous clustering algorithm due to its simplicity and speed. The $k$-means algorithm partitions the dataset according to centroids, assigning each instance to its nearest centroid.
Another type of clustering algorithm that has gained popularity in recent years are graph based clustering algorithms. The FMC algorithm [6] is one of a series of graph based methods which calculate the minimal spanning tree (MST) of a graph and select a threshold value, where edges of the MST with a weight greater than this threshold are cut. The resulting graph will be a forest with each tree corresponding to a different cluster, which performs the clustering of the original dataset. It has been shown to find clusters in a variety of different datasets and has also been shown to outperform other method in generating effective clusters [6].

The FMC algorithm has a further benefit over other graph based methods in that there are no parameters into the algorithm [6]. All of the weights of the edges in the MST are taken and clustered using the fuzzy c-means algorithm [4] searching for two clusters. As the weights represent a one dimensional dataset, there will be a 'large' cluster and a 'small' cluster. These clusters are used to determine the threshold as any weight in the 'large' cluster is cut in the original MST.

\section{THE USCAP METHODOLOGY}

The SCAP method relies on the training set of documents for each author to generate the profile. In an unsupervised learning environment such authorship classes do not exist, creating a problem for applying SCAP to these problems. To overcome this, we propose the USCAP methodology, for Unsupervised SCAP, which extends the SCAP methodology into unsupervised learning.

The USCAP methodology consists of three phases; the similarity matrix generation stage, the clustering stage and finally the ensembling stage. Firstly, the similarity between documents is calculated in much the same way that the SCAP method calculates similarity between and author and a document. A variety of parameters, specifically the choice of any preprocessing methods as well as the values for $n$ and $L$, require that this procedure is run many times for each set of parameter values resulting in many similarity matrices. Secondly, clusters are determined using the resulting similarity matrices from the first phase. This generates a list of cluster sets, where documents are clustered together in different ways from the different parameter value sets. Finally the different clusterings from the second stage are combined using an ensembling method and the results clustered for a final time to produce the final labels of the data. These three phases are now described in detail in the following subsections.

\section{A. Similarity Calculation Stage}

The USCAP methodology begins by generating the $n$ gram distribution for each document in the corpus for given values of $L$ and $n$. The key difference between the SCAP and USCAP methodologies for this step of the procedure is that the SCAP methodology calculates the overall $n$-gram distribution for each author rather, where the USCAP method calculates the $n$-gram distributions for each document. The $n$ gram distribution for a document is calculated by counting the frequency with which each $n$-gram appears in the document 
and selecting the $L$ most frequently occurring $n$-grams. This forms the simplified profile for that document. Based on previous research [7] we used $n$ values between 2 and 9 inclusive and $L$ values of 10, 50, 100, 200, 500, 1000, 1500, 2000, 3000 and None. A $L$ value of None indicates that the list of $n$-gram is not restricted in length, containing all $n$-grams in each document.

The similarity between two documents is calculated using the same method that is used in the SCAP methodology. The similarity between two documents is the size of the intersection of the simplified profile for each author, normalized by dividing by $L$, the maximum possible intersection size. The resulting value is between 0 and 1 , with 0 indicating no similarity between the documents and values close to 1 indicating a high similarity between documents. The aim for this similarity metric is that higher values indicate a higher probability that two documents are authored by the same person or group.

\section{B. Clustering Stage}

With the similarity matrices created as part of the first phase, the USCAP methodology now generates a partitioning of these documents using a clustering method. For this research we use the Fuzzy c-means MST Clustering algorithm (FMC), as described in section II-C. In this phase, the FMC algorithm is run on each of the similarity matrices calculated in phase 1 , and the resulting clusters for each are saved to be ensembles in the next phase.

\section{Ensembling Stage}

The result from phase 2 will be a large collection of different clusterings of the original dataset. Ideally there would be no difference between the clusterings from each phase 2 on each of the similarity matrices from phase 1 . In practice there will be many small differences and also a few outlying clusterings which differ substantially. To generate a final clustering of the data the ensembling. This procedure is based on a previous work [10] which used evidence accumulation which accumulates evidence in a co-association matrix (defined below) and clusters using this new matrix as a similarity measure using hierarchical clustering. The approach in this paper is the same, except that the procedure used here is based on the FMC algorithm for clustering the co-association matrix.

The procedure begins by calculating the co-association matrix $C$, where $C_{i j}$ is the percentage of phase 2 clusterings in which document $i$ and document $j$ are clustered together. This forms a similarity matrix, which is then clustered using the FMC procedure outlined in subsection III-B.

The resulting phase 3 clustering is then evaluated using the Normalized Mutual Information (NMI) score to measure the stability of the clustering from phase 2. Mutual Information is a measure comparing the amount of information shared between two series, in this case clustering labels. A high NMI score indicates a high stability in the phase 2 clustering and a high correlation to the phase 3 clustering. A low NMI score indicates that the phase 2 clustering had a lot of variation and there is little correlation to the phase 3 clustering, indicating that the methodology does not reliably determine authorship.

\section{Evaluation}

Evaluation of the results will occur in three stages. The first is the evaluation of the cluster density using the silhouette coefficient. The second is the verification using the single $U R L$ assumption described below. Finally, the clusters will be evaluated through their attacks over time. Each of these three stages will be described below.

The silhouette coefficient is a measure of cluster density and separation [28]. A high silhouette coefficient correlates to dense clusters that are well separated, while a low silhouette coefficient indicates highly overlapping clusters. The silhouette coefficient for an instance $i$ is composed of two terms. The first is the mean intra-cluster distance, $a_{i}$, which is the mean distance to each other point in the same cluster as instance $i$. The second is the mean inter-cluster distance, $b_{i}$, which is the mean distance to each other point in the nearest cluster. The silhouette coefficient for point $i$ is given as $s_{i}=\frac{b_{i}-a_{i}}{\max \left(a_{i}, b_{i}\right)}$.

The silhouette coefficient is also given for each cluster, as the mean of the silhouette coefficient of each point in that cluster. Finally, the overall silhouette coefficient is the mean of the silhouette coefficient for all points in the dataset. To evaluate a model through the silhouette coefficient, the overall value is given as well as evaluating each cluster. In some models there are well defined clusters along with overlapping clusters, in which case the cluster's silhouette coefficient will

The second form of evaluation uses the assumption that each URL associated with a phishing website should belong to a single group or campaign. In the dataset described in subsection I-B, the URL that each content was hosted on was collected and recorded along with the phishing website content. Using this data along with our assumption, the accuracy of a model can be determined by determining the number of times two phishing webpage contents share the same URL. Ideally, two contents should be clustered together each time two contents share the same URL.

Finally, the third form of evaluation is an analysis of the nature of the attacks per cluster over time. It could be expected that attacks from different clusters have little to no relationship to each other temporally. An exploratory analysis is performed on the resulting clusters using their temporal time patterns. The analysis will consider the euclidean distance between the attacks per week from each cluster. The vectors will each be normalized by clusters, with the week of the most attacks by a given cluster given a value of 1.0 and each other week having a linear proportion of that amount. Clusters with a very low distance through this method are likely to have similar authorship, as their attack patterns match closely based on time.

\section{RESULTS}

Applying the USCAP methodology to the dataset resulted in 19 clusters of authorship being discovered. The cluster sizes and labels are given in Table I. From these results, we can see 


\begin{tabular}{|r||r|l|}
\hline$\#$ & Size & $S$ \\
\hline 0 & 338 & 0.86 \\
1 & 11 & 0.86 \\
2 & 20 & 0.95 \\
3 & 154 & 0.97 \\
4 & 9 & 0.91 \\
5 & 8 & 0.93 \\
6 & 18 & 0.88 \\
7 & 8 & 0.86 \\
8 & 15 & 0.90 \\
9 & 22 & 0.87 \\
\hline 10 & 6 & 0.91 \\
11 & 7 & 0.91 \\
12 & 25 & 0.90 \\
13 & 24 & 0.92 \\
14 & 6 & 0.85 \\
15 & 7 & 0.81 \\
16 & 6 & 0.89 \\
17 & 8 & 0.89 \\
18 & 6 & 0.87 \\
19 & 6 & 0.77 \\
\hline All & 696 & 0.89 \\
\hline
\end{tabular}

TABLE I

Cluster SIZES ALONG WITH THEIR SILHOUETTE COEFFICIENT $(S)$.

that there are two major clusters; clusters 0 and 3 . Following these, there are four more clusters at or above 20 members; clusters 2, 9, 12 and 13. There are three more with membership above 10 members; clusters 1, 6 and 8. Finally, the other 11 clusters had between 6 and 9 members each. In our results, we considered any 'cluster' 5 or fewer members to be noise, of which there were 78 instances.

Most of the clusters shown in Table I are well defined, with only one cluster having a silhouette coefficient of less than 0.80 and most being above 0.85 . This indicates that the distance matrix produces dense, well separated clusters. This can be further shown in Figure 1, which shows clusters defined by dark blue blocks along the bottom left to top right diagonal. Values in each of the blocks on the diagonal are the distance between webpages in the same cluster and should tend to be dark blue. Values off the diagonal are the distances between webpages from different clusters and should tend to be more red in color. Figure 1 shows a strong tendency towards red off the diagonal, with only a few clusters indicating a lower cross-cluster distance.

Overall, the mean NMI between each of the individual clusterings and the final ensemble was 0.7685 with a standard deviation of 0.0346 . This corresponds to an average $85.9 \%$ of all decisions being the same between the clusterings and final ensemble. The two decisions that are being made are for each $i$ and $j$, that webpages $i$ and $j$ should, or should not, be clustered together. Overall, $85.9 \%$ indicates a strong correlation, despite the variance in the individual clusterings.

In performing the site based validation, an accuracy of $90.23 \%$ is achieved, 240 out of 266 decisions agree with the single URL assumption (Subsection III-D). Of the 26 errors, there were 20 different URLs and 7 clusters affected. Twelve of the URLs from cluster 8 have been also labeled in cluster 9 or vice versa, indicating that these two clusters are strongly related. The rest of the errors are single occurrences, unlikely to represent an overlapping of two clusters.

The attacks from each cluster are shown over time in Figure $2^{1}$. This figure shows each cluster along the $y$ axis and time along the $x$ axis. Darker reds indicate more attacks in that period of time, while lighter reds indicate fewer attacks. Each cluster's attack numbers have been normalized, where a dark red value of 1.0 indicates the time period which contained the most attacks from that cluster. Evaluation of this figure indicates that some of the clusters may be related. Clusters 8 and 9 have a nearly identical pattern in the same time period, indicating that they could be related. Combined with the URL based evaluation, which saw 12 of the 20 errors occurring as a mis-match between these clusters, there is strong evidence for this link. A similar example of this is between clusters 17 and 18 , including possibly cluster 15 .

Of the 20 clusters, there just 8 pairs of clusters with a euclidean distance of less than or equal to 1.0. Clusters 8 and 9 had a distance of 0.0 , with cluster 7 having a distance of 0.3 from each of these. Clusters 17 and 18 had a distance of 0.0 from each other and a distance of 0.2 from cluster 15 each. Cluster 16 had a distance of 1.0 to cluster 15 (however, a distance of 1.1 from clusters 17 and 18). Finally, clusters 3 and 5 had a distance of 0.4 from each other. This strongly correlates with what is visually available in Figure 2.

\section{CONCLUSIONS}

In this paper, a methodology for the unsupervised and automated clustering of phishing websites into campaigns was presented. The USCAP methodology extends a previous methodology to become unsupervised and applicable for cybercrime settings where labeled datasets are often hard to come across. The phishing websites are first clustered using the Local $n$-grams method with a variety of parameters. After this, a similarity matrix is created composed of the co-association between websites within each of these initial clusterings. Finally, the final clusters to be achieved using the FMC algorithm on the similarity matrix. The resulting clustering was highly distinctive, with an overall silhouette coefficient of 0.89 , and also highly correlated to each of the initial clusterings, with an overall NMI of 0.7685 corresponding to approximately $85.9 \%$ of decisions in agreement.

From the USCAP results, the size and scope of different phishing campaigns is reported on. It was found that one campaign found accounts for $43.22 \%$ of all phishing attacks, while another campaign accounts for $19.69 \%$ of attacks in our sample. There were 7 groups with medium sized campaigns, between 10 and 30 attacks in our sample each. There were 78 attacks, $9.97 \%$, that were not assigned a category and a further 77 attacks, 9.85\%, that were assigned to 'small' campaigns.

Evaluation of the clusters indicates that there are some campaigns that are likely to be related. An example of this is clusters 8 and 9 which are the most common clusters for

\footnotetext{
${ }^{1}$ The time axes on figure 2 is unlabeled, on request by the owners of the data. No alteration to the time axes has been made and it remains a linear scale.
} 


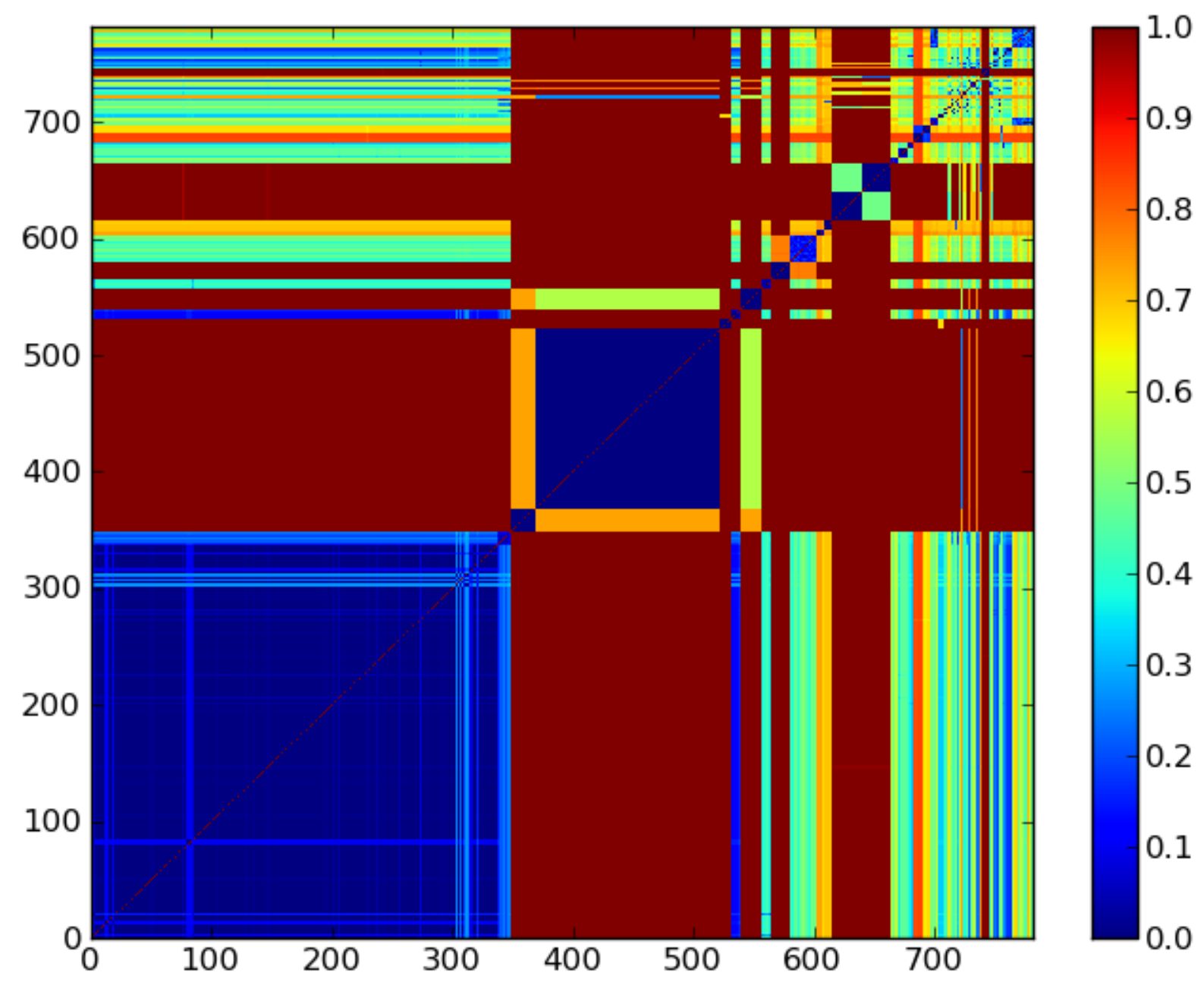

Fig. 1. Matrix visualization of the phishing websites, reordered to group clusters together along each axis. Color is the distance between the two websites.

URL based validation errors and have an almost identical similar time-attack pattern. This indicates that the clusters discovered are indicative of different campaigns from the same author. These campaigns could be linked in further research to become clusters based on complete authorship, where all attacks in a single cluster are by a single author and all attacks by a single author are in one cluster. It is possible through future investigations, that more information about these attacks can be discovered, leading to a more complete picture of the overall phishing structure.

The extensions to this work involve collecting campaigns into clusters of authorship as noted above and also applying this technique to other types of cybercrime. Cybercrime is a prime candidate for unsupervised authorship analysis work, as labeled datasets are often not available. Further to this, many cybercriminals hide behind the anonymity of the In- ternet, making it difficult to directly attribute attacks, while authorship analysis could instead attribute indirectly through methodologies such as USCAP.

\section{ACKNOWLEDGMENTS}

This research was funded by the State Government of Victoria, IBM, Westpac, the Australian Federal Police and the University of Ballarat.

\section{REFERENCES}

[1] A. Abbasi and H. Chen. Applying authorship analysis to extremist-group web forum messages. IEEE Intelligent Systems, 20(5):67-75, 2005.

[2] S. Abu-Nimeh, D. Nappa, X. Wang, and S. Nair. A comparison of machine learning techniques for phishing detection. In eCrime '07: Proceedings of the Anti-Phishing Working Group's 2nd annual eCrime Researchers Summit, pages 60-69, New York, NY, USA, 2007. ACM.

[3] R. Basnet, S. Mukkamala, and A. H. Sung. Detection of phishing attacks: A machine learning approach. Soft Computing Applications in Industry, 2008. 


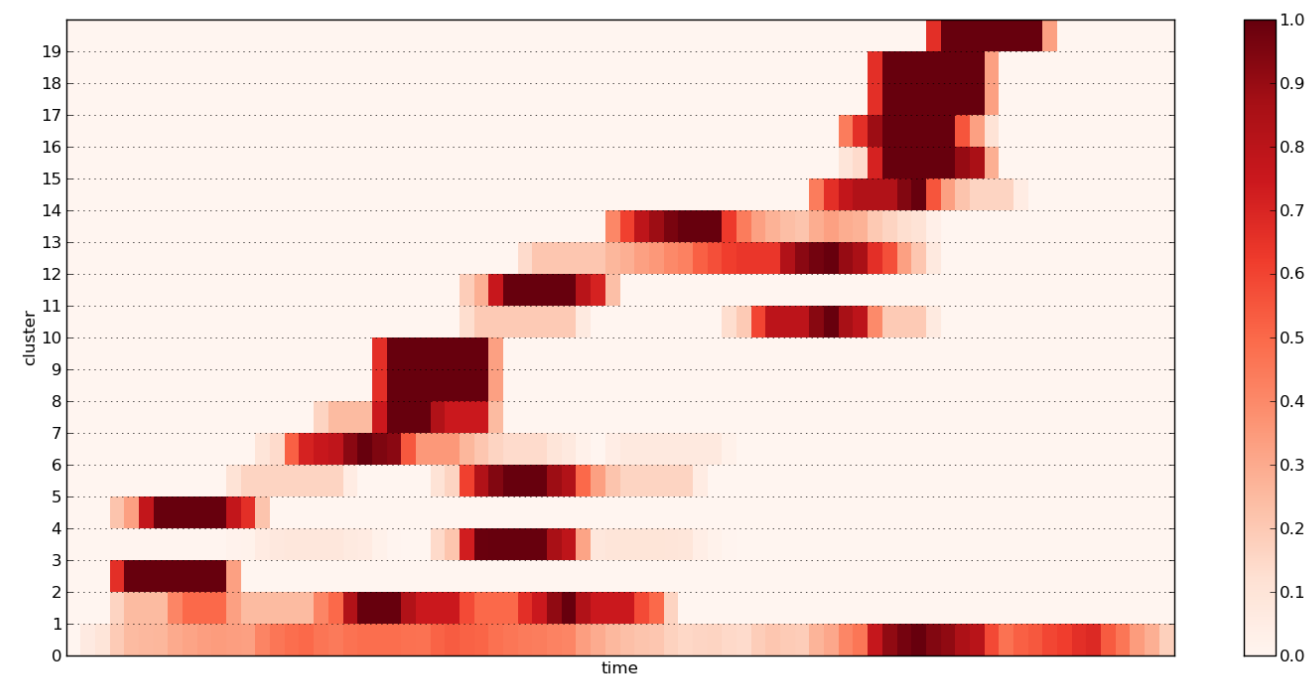

Fig. 2. Visualization of each cluster's attacks over time. Darker reds indicate more attacks, and each cluster's row has been normalized for attacks only from that cluster.

[4] J. Bezdek, J. Keller, R. Krisnapuram, and N. Pal. Fuzzy models and algorithms for pattern recognition and image processing. Kluwer Academic Publishers, 1999.

[5] M. Cova, C. Kruegel, and G. Vigna. There is no free phish: An analysis of "free" and live phishing kits. 2nd USENIX Workshop on Offensive TEchnologies, 1:1, 2008.

[6] P. Foggia, G. Percannella, C. Sansone, and M. Vento. A graph-based clustering method and its applications. Advances in Brain, Vision, and Artificial Intelligence, pages 277-287, 2007.

[7] G. Frantzeskou, E. Stamatatos, S. Gritzalis, and C. E. Chaski. Identifying authorship by byte-level n-grams: The source code author profile (scap) method. Int. Journal of Digital Evidence, 6(1), 2007.

[8] G. Frantzeskou, E. Stamatatos, S. Gritzalis, and S. Katsikas. Effective identification of source code authors using byte-level information. In ICSE '06: Proceedings of the 28th international conference on Software engineering, pages 893-896, New York, NY, USA, 2006. ACM.

[9] G. Frantzeskou, E. Stamatatos, S. Gritzalis, and S. Katsikas. Source code author identification based on n-gram author profiles. Artificial Intelligence Applications and Innovations, Volume 204/2006:508-515, 2006.

[10] A. L. N. Fred and A. K. Jain. Robust data clustering. Computer Vision and Pattern Recognition, IEEE Computer Society Conference on, 2:128, 2003.

[11] A. Gray, P. Sallis, and S. MacDonell. IDENTIFIED (Integrated Dictionary-based Extraction ofNon-language-dependent Token Information for Forensic Identification, Examination, and Discrimination): a dictionary-based system forextracting source code metrics for software forensics. pages 252-259, 1998.

[12] J. A. Hartigan and M. A. Wong. A K-means clustering algorithm. Applied Statistics, 28:100-108, 1979.

[13] V. Keselj, F. Peng, N. Cercone, and C. Thomas. N-gram-based author profiles for authorship attribution. In Proc. Pacific Association for Computational Linguistics, 2003.

[14] R. Kilgour, A. Gray, P. Sallis, and S. MacDonell. A fuzzy logic approach to computer software source code authorship analysis. International Conference on Neural Information Processing and Intelligent Information Systems, pages 865-868, 1997.

[15] Y.-G. Kim, S. Cho, J.-S. Lee, M.-S. Lee, I. Kim, and S. Kim. Method for evaluating the security risk of a website against phishing attacks. Intelligence and Security Informatics, 5075:2131, 2008.

[16] I. Krsul and E. H. Spafford. Authorship analysis: Identifying the author of a program. Computers \& Security, 16(3):233-257, 1997.

[17] R. Layton, S. Brown, and P. Watters. Using differencing to increase distinctiveness for phishing website clustering. Proceedings of the Symposia and Workshops on Ubiquitous, Autonomic and Trusted Computing, July 2009.

[18] R. Layton and P. Watters. Determining provenance in phishing websites using automated conceptual analysis. In eCrime Researchers Summit 2009, Tacoma, WA, USA, 102009.

[19] J. Ma, G. Teng, Y. Zhang, Y. Li, and Y. Li. A cybercrime forensic method for chinese web information authorship analysis. Intelligence and Security Informatics, pages 14-24, 2009.

[20] L. Ma, B. Ofoghi, P. Watters, and S. Brown. Detecting phishing emails using hybrid features. Ubiquitous, Autonomic and Trusted Computing, Symposia and Workshops on, 0:493-497, 2009.

[21] L. Ma, R. Torney, P. Watters, and S. Brown. Automatically generating classifier for phishing email prediction. Parallel Architectures, Algorithms, and Networks, International Symposium on, 0:779-783, 2009.

[22] S. MacDonell, A. Gray, G. MacLennan, and P. Sallis. Software forensics for discriminating between program authors using case-based reasoning, feed forward neural networks and multiple discriminant analysis. 1 , 1999.

[23] S. McCombie, P. Watters, A. Ng, and B. Watson. Forensic characteristic of phishing - petty theft or organized crime? In J. Cordeiro, J. Filipe, and S. Hammoudi, editors, WEBIST (1), pages 149-157. INSTICC Press, 2008.

[24] D. K. McGrath and M. Gupta. Behind phishing: an examination of phisher modi operandi. In LEET'08: Proceedings of the 1st Usenix Workshop on Large-Scale Exploits and Emergent Threats, pages 1-8, Berkeley, CA, USA, 2008. USENIX Association.

[25] T. Moore and R. Clayton. Examining the impact of website take-down on phishing. In eCrime '07: Proceedings of the anti-phishing working groups 2nd annual eCrime researchers summit, pages 1-13, New York, NY, USA, 2007. ACM.

[26] F. Mosteller and D. L. Wallace. Inference in an authorship problem. Journal of the American Statistical Association, 58(302):275-309, 1963.

[27] P. Oman and C. Cook. Programming style authorship analysis. In Proceedings of the 17th conference on ACM Annual Computer Science Conference, pages 320-326. ACM New York, NY, USA, 1989.

[28] P. Rousseeuw. Silhouettes: a graphical aid to the interpretation and validation of cluster analysis. Journal of computational and applied mathematics, 20(1):53-65, 1987.

[29] E. Stamatatos. A survey of modern authorship attribution methods. Journal of the American Society for Information Science and Technology, 60(3), 2009. 
[30] R. Thomas and J. Martin. The underground economy: priceless. The USENIX Magazine, December, 00:2006-12, 2006.

[31] J. Toolan, F. Carthy. Phishing detection using classifier ensembles. pages $1-9,2009$.

[32] T. Urvoy, E. Chauveau, P. Filoche, and T. Lavergne. Tracking web spam with html style similarities. ACM Trans. Web, 2(1):1-28, 2008.

[33] T. Urvoy, T. Lavergne, and P. Filoche. Tracking web spam with hidden style similarity. AIRWeb 2006 Program, 29:25, 2006.

[34] R. Zheng, J. Li, H. Chen, and Z. Huang. A framework for authorship identification of online messages: Writing-style features and classification techniques. Journal of the American Society for Information Science and Technology, 57(3):378-393, 2005. 